\title{
Fabian Schwitter
}

\section{Ulf Stolterfohts fachsprachen}

\section{Die eigentümliche Unpersönlichkeit von Form und Erkenntnis}

Das Wahre ist das Ganze. (G. W. F. Hegel)

What I shall have to say here is neither difficult nor contentious; the only merit I should like to claim for it is that of being true; at least in parts. (John L. Austin)

>Ich « sei derjenige Index oder derjenige deiktische Ausdruck - und es gibt weitere deiktische Ausdrücke -, welcher die Sprechinstanz markiere. So sagt das Linguistische Wörterbuch beim Stichwort >deiktische Ausdrücke`:

Die Person-Kategorie ich bildet meist das Zentrum bzw. den Nullpunkt des deiktischen Systems. (Lewandowski 1990, 205)

Es scheint auf der Hand zu liegen, dass ein Ich als »Nullpunkt« eines Kommunikationssystems, das heißt einer Sprechsituation, unabdingbar ist - sofern Kommunikation einen Informationstransfer von einer Instanz zu einer anderen (oder sogar mehreren anderen) bedeutet. Das Ich jedenfalls ermöglicht die Zuschreibung einer Äußerung einer bestimmten Sprechinstanz - meist gehen wir davon aus, dass es sich dabei um einen Menschen handelt. Die Äußerung ist verbunden mit einer Person: Ein Wind mit trocken- sodann feuchtadiabatischem Temperaturabfall im luvseitigen Steigen und anschließendem trockenadiabatischem Temperaturanstieg im leeseitigen Fallen und teilweiser überkritischer Geschwindigkeit unter Berücksichtigung der gap dynamic beim Passieren von topografischen Lücken bereitet mir Kopfschmerzen. Ich, in diesem Fall als Schreibender, bin Teil der Äußerung, und du magst dir jetzt denken, ich sei ein Angeber, der mit fachsprachlichem Jargon prahlt - eben, weil ich als namentlich genannter Schreibender Teil der Äußerung bin.

Diese Äußerung hingegen - Es gibt einen Wind mit trocken- sodann feuchtadiabatischem Temperaturabfall im luvseitigen Steigen und anschließendem trockenadiabatischem Temperaturanstieg im leeseitigen Fallen und teilweiser überkritischer Geschwindigkeit unter Berücksichtigung der gap dynamic beim Passieren von topografischen Lücken - braucht nicht eindeutig zugeschrieben zu werden. Hinter der Äußerung könnte irgendeine Person stehen: eine Meteorologin vielleicht oder sogar ein Wettersatellit, sofern er über die nötigen technischen Mittel 
verfügte, solches zu äußern. Ich wiederum, der diese Äußerung niedergeschrieben hat, könnte lediglich Versatzstücke aus einem bekannten Online-Lexikon wiedergeben. Und es hätte im Übrigen auch keinen Sinn, eine solche Äußerung eindeutig zuzuschreiben (es sei denn den Sinn, die sich äußernde Person als Lügner zu entlarven). Entweder die Äußerung ist in sich stimmig und trifft (in einem bestimmten Moment) zu oder eben nicht. Ob also hin und wieder Föhnlagen herrschen und die Aussage folglich wahr ist, bleibt dir zur Prüfung überlassen und ist unabhängig von mir, der diese Äußerung von sich gegeben hat.

Sofern ein Ich vorliegt, können wir davon ausgehen zu wissen, dass jemand spricht. Die Äußerung ist zuschreibbar. Wenigstens können wir den zeitlichen und räumlichen Punkt der sprechenden Person im Koordinatensystem der Welt bestimmen, auch wenn wir ihren Namen oder Beruf nicht kennen. Wir wissen, es ist diese Person: hier und jetzt. Und später werden wir darauf beharren, dass ein bestimmter Wissenschaftler diese Ausführungen geschrieben hat, und ihn dafür zur Verantwortung ziehen. (Bezeichnenderweise bleibt selbst in wissenschaftlichen Texten mit ihren vermeintlich objektiven und personenunabhängigen Aussagen die sich äußernde Instanz relevant. Schließlich gibt sich das Ich in Form des Autorennamens zu erkennen, obwohl der Autorennamen hinsichtlich der Wahrheit einer wissenschaftlichen Äußerung nichts zur Sache tut.) Soweit scheint die Situation übersichtlich - übersichtlicher jedenfalls, nachdem Sprachphilosophen wie John Austin oder Ludwig Wittgenstein sich des Problems angenommen haben.

Etwas komplizierter scheint der Fall mit dem sogenannten >lyrischen Ich $\mathrm{zu}$ liegen, von dem als leichter Präzisierung dem Reallexikon der deutschen Literaturwissenschaft gemäß gesprochen wird, wenn von Gedichten die Rede ist. Und auch Austin schränkt seine Sprechakttheorie schon früh ein: »I must not be joking, for example, nor writing a poem. "(Austin 1976, 9) Wenn es aber doch um ein Gedicht und das Ich geht? Harald Fricke und Peter Stocker (2000) sagen im Reallexikon der deutschen Literaturwissenschaft zum lyrischen Ich, es sei »das Subjekt von Gedichten ohne Rollenfiktion«. Auch wenn beide festhalten, dass das lyrische Ich »unabhängig von seiner grammatischen Form « sei, gehe ich vorderhand vom explizit erwähnten Pronomen >ich` aus, um die Situation nicht unnötig zu verkomplizieren und die Übersicht zu verlieren. So verstanden markiert dieses Ich eine nicht näher bestimmte Sprechinstanz - und diese ist schließlich unabdingbar. Gemäß dem Reallexikon bezeichnet das lyrische Ich »eine poetische Leerstelle, die zur imaginativen Füllung ebenso einlädt wie zur persönlichen Identifikation." (Fricke und Stocker 2000, 509)

Im Fall eines Gedichts, in dem die Sprechinstanz durch das Pronomen bzw. den Index sich aber Sprechinstanzen auszumachen bzw. die Kommunikationssituation scheint klar: 
1. Sofern das Gedicht bloß als Vermittler betrachtet wird, markiert das Ich eine mehr oder weniger genau bestimmte Sprechinstanz bzw. Person (Alter? Geschlecht? Beruf? Wünsche? Enttäuschungen?), die sich oder etwas mittels des Gedichts den Lesenden näherbringt.

2. Sofern das Gedicht selbstreferentiell gelesen wird, gehört das Ich zum Gedicht selbst. Das Gedicht spricht - in einer poetologischen Wendung - von sich selbst.

Was ist jedoch, wenn kein Ich auszumachen ist? Wenn das Wort \Ich` im Gedicht oder sogar in mehreren Gedichten schlicht nicht auftaucht? Und falls doch, es bloß als weitere Instanz in einer Dialogsituation oder gar eher zufällig unter anderen Dingen auftaucht? Wer spricht dann? - Gut, ich muss ehrlich und philologisch genau genug sein (man wird jenen Wissenschaftler schließlich zur Verantwortung ziehen), einzugestehen, dass das Ich doch an einigen Stellen in fachsprachen des Lyrikers Ulf Stolterfoht vorkommt, wenn auch nicht in allen 324 Gedichten, der bislang erschienenen Fachsprachenbände: fachsprachen I-IX (Stolterfoht 2005), fachsprachen X-XVIII (Stolterfoht 2008), ${ }^{1}$ fachsprachen XIX-XXVII (Stolterfoht 2005) und fachsprachen XXVIII-XXXVI (Stolterfoht 2009). Und dennoch möchte ich daran festhalten, dass dieses Ich im Grunde genommen irrelevant und auch kaum weiter bestimmbar ist. Eher drängt sich der Verdacht auf, dass da ganz unterschiedliche Ichs nicht nur über die genannten vier Fachsprachenbände, sondern auch innerhalb einzelner Gedichte verteilt auftreten. Wenigstens dies hoffe ich, in der Folge zeigen zu können.

Der Titel »fachsprachen« weist darauf hin, dass es im Ganzen ohnehin nicht um das individuelle Sprechen Einzelner geht. Einerseits könnten potenziell alle Sprechende sein (nach dem Erlernen der Fachsprache) bzw. werden (durch das Erlernen der Fachsprache). Andererseits dienen Fachsprachen jedoch auch - vielleicht im Sinne der Disziplin aus Michel Foucaults Ordnung des Diskurses ${ }^{2}$ - zur

12005 bzw. 2008 erschienen jeweils Zweitauflagen der ursprünglich 1998 bzw. 2002 publizierten fachsprachen I-IX und fachsprachen X-XVIII.

2 Mit einer leichten Verschiebung lässt sich hinter Foucaults Opposition von Vernünftigen und Wahnsinnigen heute durchaus auch die Opposition von Fachleuten bzw. sogenannten Experten und Laien vermuten (vgl. Foucault 1991, 11-12). Gerade die Opposition von Wahrem und Falschem in Zusammenhang mit der Opposition von Vernunft und Wahnsinn belegt allerdings auch, dass die sprechende Instanz entscheidend ist (erinnert sei an den Lügner). Die von Vornherein vorgenommene Zuschreibung des Fachpersonenstatus gibt einer gemachten Aussage Kredit. Insofern spielt das Ich im Diskurs immer wieder eine Rolle, auch wenn es sich selbst wiederum in seiner Position durch das Beherrschen der jeweiligen Fachsprache legitimiert. Person und Fachsprache legitimieren sich gegenseitig und wachen peinlich genau über ihre Einflusssphäre (vgl. Foucault 
Strukturierung eines Diskurses und damit als Ein- und Ausschlusskriterium für potenzielle Sprechende. Wer die Regeln des Diskurses oder eben die Fachsprache nicht beherrscht, ist als Sprechender oder Sprechende nicht zugelassen. - An dieser Stelle ist es, da ich - durchaus ohne Verlegenheit - aus Gründen der Textkohärenz darauf verzichtet habe, angezeigt, meine fachsprachliche Zulassung vorzuweisen. Die bislang referierte Begrifflichkeit - insbesondere das verhältnismäßig vage >lyrische Ich « - wurde von Rüdiger Zymner mit Blick auf die Begründung einer >Lyrikologie` (als Pendant zur >Narratologie`) angepasst und zugespitzt, womit zunächst einmal die Bedeutung des ১lyrischen Ichs` von einer >Instanz« zu einer »spezifischen Zeichenstruktur« verschoben und die Bezeichnung >lyrisches Ich in Konsequenz durch die Bezeichnung >Adressant $<$ ersetzt wird. ${ }^{3}$ Unter der Voraussetzung seiner Begrifflichkeit sind die fachsprachen im Ganzen als sadressantenneutral zu bezeichnen, wiewohl sie im Einzelnen vielfältig ıadressantenmarkiert` sind, wie sich noch zeigen wird (in der Folge werden zymnersche Präzisierungen mit Ausnahme der Adressanten/Adressaten-Thematik - in Klammern und Anführungsstrichen angegeben; diese resultierende Begrifflichkeitsverdoppelung trägt dem Umstand Rechnung, dass letztlich im Fall des untersuchten Gegenstandes, der fachsprachen Stolterfohts, nicht gewährleistet ist, ob es sich tatsächlich um >Lyrikı im Sinne Zymners handelt: Stolterfohts Gedichte konstituieren »ästhetische Evidenz« zwar in hohem Maß »prozedural«, allerdings muss zweifelhaft bleiben, ob sie »Sprache als Medium prozeduraler Sinngenese« exponieren, da `Sinn Bezug auf Stolterfohts Lyrik zumindest ein prekärer Begriff ist). - Fachsprachen sind, auf den ersten Blick jedenfalls, nicht allen zugänglich, was sie im Vergleich mit Alltagssprache hermetisch erscheinen lässt. Stolterfoht, mit Blick auf Oskar Pastiors Gedichtband Wechselbalg, wehrt sich zwar mit seiner Unterscheidung zwischen Schwerverständlichkeit und Unverständlichkeit im Grunde genommen gegen Hermetik, wie seine Münchner Rede zur Poesie klarstellt:

Das schwer verständliche, hermetische Gedicht fordert mich als Leser zu hermeneutischen Bestleistungen auf [...]. Wenn das schwer verständliche Gedicht das aristokratische, elitäre und hierarchische Gedicht ist, [...] dann waren diese Gedichte demokratisch und unhierarchisch. [...] Das Unverständliche dieser Gedichte war also eher ein Nicht-verstehen-müssen als ein Nicht-verstanden-werden-können oder -wollen. (Stolterfoht 2015, 11-12)

1991, 13-17 und 22-25). Besonders drastisch zeigt sich die Disziplin, die durchaus auch als Fach, wie beispielsweise Medizin, mit seiner spezifischen Sprache verstanden werden darf, in Foucaults Metapher einer Diskurspolizei: »Es ist immer möglich, dass man im Raum eines wilden Außen die Wahrheit sagt; aber im Wahren ist man nur, wenn man den Regeln einer diskursiven >Polizei< gehorcht, die man in jedem seiner Diskurse reaktivieren muss. / Die Disziplin ist ein Kontrollprinzip des Diskurses.« (Foucault 1991, 25).

3 Vgl. in diesem Band 26. 
Allerdings braucht es trotz allem einen Moment, um die anfängliche Befremdung beim Lesen der Fachsprachengedichte zu überwinden - zumindest für diejenigen Lesenden, die auf klare und einfache Bedeutungen ( $>$ Information ` oder sogar ıSinn`) aus sind. Insofern sind die Fachsprachengedichte doch hermetisch, auch wenn sie einen anderen Anspruch erheben:

deklaration des wust

zitatpfeile zuhauf, nöcher denkspeck im köcher. doch alles zu messen in gramm. anders gesagt: unser lyrikprogramm - spannend wie nannen / beklemmend wie rihm. die zimmerfluchten neuköllns dürften damit aller-

dings in weite ferne gerückt sein. haust man halt jenseits des betriebs. verfaßt - da stockt dir der atem - berichte über genagelte raben. kombiniert magische muffler mit ionischem bandmaterial »he's breeding a dwarf«. der wust-

beweis ist angetreten. jetzt wird er noch erklärt: voll-wust. not-wust. wust, der sich wie ein hunnennetz (tacitus) zusammenzieht. trug-wust. davon tonnnen auf halde. wußten sie

übrigens, daß heinrich zille in fürstenwalde hochzeit hielt? nein, das wußte ich nicht. sapir und whorf in schorfheide? nein, das wußte ich nicht. auch das ist mir vollkommen neu. (Stolterfoht 2009, 103)

Das Ich tritt im obigen Gedicht aus »fachsprachen XXXV« vielfältig auf. So vielfältig, dass es keinesfalls ausschließlich mit einem Sprecher koinzidiert, als hätten sich die Fachsprachen - mit ihrer Community von Sprechenden, die darüber wacht: wer auch immer sie sein mögen - verselbständigt. So redet eines dieser berüchtigten und vorderhand ungenannten slyrischen Ichs es zuerst sich selbst kommentiert und somit aufgespalten hat (»jetzt wird er [der Wust] noch erklärt«), direkt an und konfrontiert sie gegen Ende des Gedichts mit Fragen: »wußten sie übrigens, daß heinrich zille in fürstenwalde hochzeit hielt?« Unvermittelt taucht das Ich dann doch explizit auf - und zwar, so scheint es, ${ }^{4}$ in der Rolle der Rezipienten (oder der >Adressaten`) mit der Antwort: »nein, das wußte ich nicht.« Und weiter: »sapir und whorf in schorfheide?« Antwort: »nein,

4 Textintern ist bis zu dieser Stelle kaum ein Adressat auszumachen. Das zweite Quartett schafft mit seiner Anrede (»da stockt dir der atem«) zwar eine intime Situation, die allenfalls auf eine textinterne Dialogsituation schließen lässt. Die folgende Anrede in der Höflichkeitsform bricht allerdings mit der vorher etablierten Situation. 
das wußte ich nicht. auch das ist mir vollkommen neu.« Urplötzlich ist das Ich verschoben und das lyrische Ich genauso wie die Lesenden, als unwissende Laien (im Gegensatz zum kompetent sprechenden Fachmann) entlarvt, scheint auf einmal aus dem Diskurs seiner eigenen fachsprache herausgefallen und ausgeschlossen zu sein. Die Identifikation des explizit genannten Pronomens mit dem (vorangehenden) >Adressanten liegt als Möglichkeit insofern nahe, als Zymners Glossar festhält, in einem lyrischen Text würden der >Adressant pronomina wie >ich ` oder >wir bzw. in dialogischen Sequenzen gegebenenfalls auch durch die Personalpronomina >du oder sihr implizit markiert. Bezüglich Stolterfohts Gedicht lässt sich sagen: Wo der >Adressant ‘ klar scheint, figuriert er bloß implizit, und wo der >Adressant ` unklar scheint, steht seine explizite Markierung. Ein veritables Chaos, zumal keinerlei Markierungen (`Schriftzeichen`) wie Anführungsstriche einen Dialog deutlich machen. Der Dialog lässt sich bestenfalls durch die Struktur des Gedichts (`Text ) erschließen. Festzuhalten ist jedenfalls,

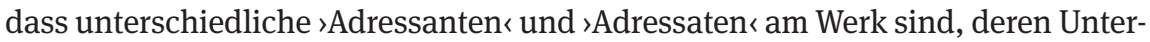
scheidung unter dem Begriff >Adressant` bestenfalls durch Zahlen gewährleistet würde: >Adressant 1 , >Adressant 2«, >Adressant $3<$... Über deren mögliche Koinzidenz ist damit noch nichts gesagt, ebenso wenig wie über die jeweilige Verteilung der Produktions- und Rezeptionsposition. Diese `Adressanten bleiben jedoch so unspezifisch, dass sich beispielsweise hinter dem >Adressanten ebenso gut eine imaginierte Instanz (১Persona`) wie der Verfasser (`Autor`) selbst verbergen könnte. Letztlich läuft dieser Sachverhalt darauf hinaus, den Adressanten durch Multiplikation zu destabilisieren.

Das - implizite oder explizite - Ich hat sich auf vielfältige Weise manifestiert. Einerseits beginnt das Gedicht, als würde da etwas von irgendeinem Individuum erzählt. Andererseits verschiebt sich das Gedicht bereits in der zweiten Zeile, sodass ein Kollektiv (»unser lyrik- / programm«) auftritt - nur um im Verlauf nicht nur zurückzukehren, jedoch verschoben (als aufgespaltenes, sich selbst kommentierendes Ich), sondern dieses Ich auch noch in zweifacher Weise als Dialoginstanz (zuerst als Produzent, dann als Rezipient) zu präsentieren.

Jedenfalls könnte ich mich nach diesem Beispiel zur Charakteristik der fachsprachen, genau wie Stolterfoht das an vielen und nicht nur der obigen Stelle (»wußten sie, dass... «; »wußten sie, dass...») macht, in einer Aufzählung ergehen - einer Aufzählung der unterschiedlichen Erscheinungsformen der Fachsprachen in ihrer Ähnlichkeit. Als erste Annäherung genügt es jedoch festzuhalten, dass die einzelnen Blöcke, pro Band je neun zu je neun Gedichten mit jeweils bestimmter Strophen- und Verszahl, immer gleich aufgebaut sind: Jede Strophe bildet ein beinahe exaktes Rechteck, dessen Verse weder metrisch definiert noch über den Reim gebunden, sondern rein graphisch-geometrisch angeordnet sind ( ‘außermetrische Lyrikı). Aus fachsprachen XXVIII-XXXVI folgende graphisch 
dargestellten Schemata der letzten drei Blöcke als Beispiele (jede dieser Fachsprachen umfasst neun Gedichte entsprechend dem jeweiligen Schema):

fachsprachen XXXIV

\begin{tabular}{|l|}
\hline 1. Zeile \\
2. Zeile \\
3. Zeile \\
4. Zeile \\
5. Zeile \\
6. Zeile \\
\hline \\
7. Zeile \\
8. Zeile \\
9. Zeile \\
10. Zeile \\
11. Zeile \\
12. Zeile \\
\hline
\end{tabular}

\begin{tabular}{|l|}
\hline 13. Zeile \\
14. Zeile \\
15. Zeile \\
16. Zeile \\
17. Zeile \\
18. Zeile \\
\hline
\end{tabular}

\begin{tabular}{|l|}
\hline 19. Zeile \\
20. Zeile \\
21. Zeile \\
22. Zeile \\
23. Zeile \\
24. Zeile \\
\hline
\end{tabular}

fachsprachen XXXV
\begin{tabular}{|l|}
\hline 1. Zeile \\
2. Zeile \\
3. Zeile \\
4. Zeile \\
\hline 5. Zeile \\
6. Zeile \\
7. Zeile \\
8. Zeile \\
\hline 9. Zeile \\
10. Zeile \\
11. Zeile \\
\hline
\end{tabular}

\begin{tabular}{|l|}
\hline 12. Zeile \\
13. Zeile \\
14. Zeile \\
\hline
\end{tabular}

fachsprachen XXXVI

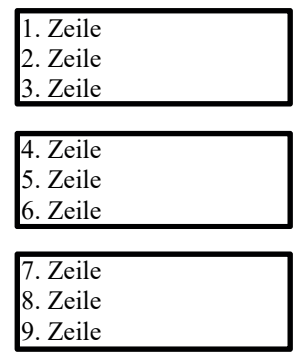

\begin{tabular}{|l|}
\hline 10. Zeile \\
11. Zeile \\
12. Zeile \\
\hline
\end{tabular}

\begin{tabular}{|l|} 
13. Zeile \\
14. Zeile \\
15. Zeile \\
\hline
\end{tabular}

\begin{tabular}{|l|}
\hline 16. Zeile \\
17. Zeile \\
18. Zeile \\
\hline
\end{tabular}

\begin{tabular}{|l|}
\hline 19. Zeile \\
20. Zeile \\
21. Zeile \\
\hline
\end{tabular}

Abb. 1: Aufbauschema der Fachsprachenbände.

Die formalen Details, dass »deklaration des wust « (genau wie die anderen acht Gedichte im Block »fachsprachen XXXV« aus fachsprachen XXVIII-XXXVI) beispielsweise an das Sonett angelehnt ist (sie bilden damit eine Ausnahme), scheinen - zu diesem Zeitpunkt jedenfalls - weniger entscheidend. Denn Fachsprachen und ihre Hermetik verlangen ganz allgemein nach einer bestimmten Darstellung, auch wenn diese - genau wie bei den fachsprachen - von Fall zu Fall leicht variiert. Das ließe sich mit Blick auf akademische Gepflogenheiten leicht zeigen, ist doch jedes Fach nicht nur stolz auf seinen Fachjargon, sondern auch auf eine bestimmte, in Nuancen von anderen abweichende Darstellungsweise ihrer Resultate. Am deutlichsten zeigt sich das beim unterschiedlichen Handhaben von Zitaten und Fußnoten in Seminararbeiten, die doch nie für den Druck bestimmt waren. Peinlich genau wird auch dort darüber gewacht, dass die jeweilig verlangte Darstellungsweise von den Studierenden eingehalten wird. 
Das klingt nun alles recht schulmeisterlich und allzu pedantisch trocken. Alles in allem eine recht freudlose Angelegenheit. Tatsächlich verhält es sich jedoch mit den fachsprachen (und übrigens auch mit den Fachsprachen) nicht so. Theodor Ickler, und es dürfte sich um den bekannten Gegner der Rechtschreibereform handeln, wie mir ein Online-Lexikon verrät - macht jedenfalls auf der Rückseite des ersten Fachsprachenbandes fachsprachen I-IX dankenswerterweise darauf aufmerksam - und das sicher ganz im Sinne Stolterfohts, gibt er sich in seiner Münchner Rede zur Poesie doch als »Experte für Euphorie« (Stolterfoht 2015, 7) zu erkennen:

Tatsächlich gebraucht der Fachmann die von ihm mitsamt dem Fach beherrschte Fachsprache oft mit einer unleugbaren `Funktionslustı, etwa so wie man ein gut beherrschtes Musikinstrument spielt. Das muß mit Imponierlust und Exklusivität nichts zu tun haben. Man unterstellt das viel zu oft und unbedenklich, als gäbe es gar nicht so etwas Unschuldiges wie die Freude am erkennen [sic!] und am wohlgelungenen Meistern der Materie. (Stolterfoht 2005)

Nicht nur kommen aber in den fachsprachen, die ebenso lustvoll, wie sie geschrieben wurden, auch gelesen werden sollen, Fachsprachen im weitesten Sinn zur Darstellung, sondern die Lyrik selbst erweist sich als Fachsprache. Neben einer Reihe von Logikern nur schon in fachsprachen XXVIII-XXXVI beispielsweise »meinong« (Stolterfoht 2009, 94), »frege« (Stolterfoht 2009, 32, 94 und 96) oder "searle« (Stolterfoht 2009, 94) - treten auch bekannte Namen aus der Lyriktradition auf: »czernin« (Stolterfoht 2009, 18), »mayröcker« (Stolterfoht 2009, 114), »jandl. hölderlin.« (Stolterfoht 2009, 115), »heißenbüttel« (Stolterfoht 2009, 117), »mörike« (Stolterfoht 2009, 118), »benn« (Stolterfoht 2009, 119). Logische Terminologie - »ich habe diese aussage eigenhändig formalisiert.« (Stolterfoht 2009, 66) oder »so knallig schallt der modus ponens. [/] und: F ! das ist das fregesche behauptungszeichen. «(Stolterfoht 2009, 94) - steht neben Dialekten und Fremdsprachen: vom mehrfach erwähnten »sasse« (Stolterfoht 2009, 23 und 34), das doppelt fachsprachlich in der ostniederdeutschen Jägersprache Hasenlager meint, über die schwäbische »fasnet« (Stolterfoht 2009, 30) bis hin zum Niederländischen oder seiner flämischen Varietät sinnigerweise im Gedicht babel „verstaat u mij?/ja, als u niet te vlug spreekt!« (Stolterfoht 2009, 65). Und immer wieder die Lyriktradition: »fachsprachen XXXV« (Stolterfoht 2009, 101-109) als Anlehnung ans Sonett, der »versbruch« (Stolterfoht 2009, 97) als ästhetisches Prinzip oder Bestimmung des Gedichts schlechthin, die Erwähnung von »hombroich« (Stolterfoht 2009, 113) mit der Raketenstation, wo unter anderen Thomas Kling lebte. Darüber hinaus als mögliche Erklärung für die Programmatik der fachsprachen, wonach dem Gedicht durchaus eine bestimmte Hermetik eigen und es folglich nicht allen zugänglich ist, eine Celan-Referenz: "gedicht als losungswort» 
(Stolterfoht 2009, 93). Stolterfoht zitiert indirekt das »No pasarán« der Widerstandskämpfer im spanischen Bürgerkrieg, das Celan unter anderem im Gedicht »Schibboleth« (Celan 2005, 83) aufgegriffen hat. Und ein weiteres Beispiel ist die Anspielung auf Hölderlins Gedicht »Der Winkel von Hahrdt. « (Hölderlin 2000, 758) in der Schlusszeile des Gedichts »48/49«: »gedenkst du noch manchmal des winkels bei hardt « (Stolterfoht 2009, 115). Alles in allem - und die oben angeführten Beispiele sind bloß ein Bruchteil - bilden die fachsprachen und in der Folge notgedrungen auch die Fachsprachen ein recht unüberschaubares Hit-Potpourri.

Wie Stolterfoht sich also Versatzstücken aus Fachsprachen bedient, so bediene ich mich Versatzstücken aus fachsprachen, um den fachsprachen - und vielleicht auch den Fachsprachen - auf die Spur zu kommen. Wenig anderes bleibt mir übrig angesichts der programmatischen Unverständlichkeit dieser fachsprachen und angesichts des unter allen Umständen beschränkten Raums. Schließlich geht es, und das unterstreichen die mittlerweile vier erschienenen Bände (und ein fünfter und sechster sind meines Wissens zumindest in der Konzeptionsphase, wenn nicht schon druckreif in der Schublade ${ }^{5}$ ), um die fachsprachen und keinesfalls nur um einzelne Gedichte. Das scheint mir angesichts ihrer idiosynkratischen Erscheinung (১typographisches Dispositiv $\iota$ ) auf der Hand zu liegen. Denn weshalb sollten alle Fachsprachenbände gleich strukturiert sein, wenn es bloß um einzelne Gedichte ginge? So bin ich gezwungen, einerseits eine allgemeine Annäherung an die Fachsprachen im Ganzen zu versuchen und andererseits diese Annäherung aufgrund des beschränkten Rahmens eines wissenschaftlichen Artikels anhand einzelner, soweit überschaubarer Versatzstücke - das heißt in diesem Fall anhand des vierten Fachsprachenbandes und des Gedichts »deklaration des wust « - deutlich zu machen. Insofern erklärt sich auch, dass die einleitend angedeutete Systematik zur 〉Deixis` und zum 〉lyrischen Ich <einesfalls den Anspruch erheben, das Thema erschöpfend dargelegt zu haben, sondern dass sie lediglich ein möglicher Ansatzpunkt sind, von dem aus gewissermaßen ex negativo eine Annäherung an die fachsprachen möglich ist.

An dieser Stelle bietet sich vielleicht eine Bemerkung zum Verhältnis der fachsprachen zu den Fachsprachen an: Sie stehen in einer chiastischen Verschränkung zueinander. Die wissenschaftliche Form, das heißt die literaturwissenschaftliche Fachsprache, zitiert lyrische Sprache in einem argumentativen Kontext. Die lyrische Form, das heißt die fachsprachen, zitiert argumentativ-wissenschaftliche Sprache in einem lyrischen Kontext. Über die daraus folgenden Implikationen wäre nachzudenken - sicher macht sich in dieser Verschränkung jedoch die gegenseitige Angewiesenheit bemerkbar. Beide treffen sich vielleicht in ihrem Interesse

5 Das zumindest hat mir Ulf am 30. Mai 2016 bei einem Glas Bier in Zürich erzählt. 
an Erkenntnis. Doch im Fall der Wissenschaft gilt das Erkenntnisinteresse einem Gegenstand, von dem gesprochen wird, sodass das Medium unweigerlich im Hintergrund bleiben muss, während in der Lyrik - zumindest derjenigen Stolterfohts das Erkenntnisinteresse dem Medium gilt, während der Gegenstand, von dem trotz allem unweigerlich gesprochen werden muss, im Hintergrund bleibt.

Es geht also in den Fachsprachen genauso wenig wie in den fachsprachen um einzelne Texte, einzelne Sprechende oder überhaupt sonst um etwas Einzelnes - allerhöchstens um gezielt Vereinzeltes: Versatzstücke aus unterschiedlichen Fachsprachen kompiliert zu Gedichten. Das dürfte anhand der obigen Aufzählung von Namen deutlich genug geworden sein. Worum kann es aber dann gehen? Das entscheidende Element, das sich bis dahin in der Auseinandersetzung mit Fachsprachen abgezeichnet hat, ist die Wiederholbarkeit der fachsprachen unabhängig von einer bestimmten - wissenschaftlichen ebenso wie lyrischen - Sprechinstanz oder einem bestimmten >Adressanten<. Der lakonische Schluss des Gedichts «50/51" aus dem vierten Fachsprachenband scheint mir das überdeutlich zu machen: »das, was ich wirklich will, das macht bereits hans thill." (Stolterfoht 2009, 116) Sprechende einer Fachsprache sind meist nicht die ersten, die sich einer Fachsprache bedienen. Für Fachsprachen charakteristische terminologische Prägungen gelingen nur in den seltensten Fällen. Meist werden Terminologien von den Sprechenden einfach wiederholt ${ }^{6}$ und auf neue Gegenstände und Kontexte angewendet. Das zwingt auch fachsprachen dazu, in einer je spezifischen Weise zu erscheinen. Die erwiesenermaßen auftretenden Koinzidenzen in der Darstellung (`typographisches Dispositiv`) zwischen unterschiedlichen Blöcken über die einzelnen Bände hinweg wären jetzt zu prüfen: Handelt es sich bei gleich erscheinenden Gedichtblöcken tatsächlich um dieselbe Fachsprache? ${ }^{7}$ Ob darüber hinaus mit dem Ich in der vorher zitierten Zeile Stolterfohts, ein belie-

6 Foucault, durchaus jemand, der Terminologien geprägt hat, beginnt seine Ordnung des Diskurses mit dem emphatischen Wunsch nach Verdoppelung: »Ich hätte gewünscht, dass es hinter mir eine Stimme gäbe, die schon seit langem das Wort ergriffen hätte und im vorhinein alles, was ich sage, verdoppelte. (Foucault 1991, 9) Das Glück eines genuin Neuen ist untrennbar mit Verantwortung verbunden. Es mag eine paradoxe Pointe sein, dass sich je nach Position die Wünsche in ihr Gegenteil verkehren. Wer den Diskurs prägt, mag im Wissen um das Gewaltsame dieser Prägung wünschen, er würde bloß wiederholen (um vielleicht seiner Vorgänger, im Falle Foucaults Jean Hippolyte, angemessen zu gedenken). Wer den Diskurs nicht prägt, mag wünschen, doch endlich einmal etwas Neues - Persönliches - einführen zu können.

7 Am häufigsten (nämlich siebenmal verteilt über alle vier bislang erschienen Fachsprachenbände) taucht das Format `sechsmal vier ‘ auf, das heißt sechs vierzeilige Strophen: zweimal in fachsprachen I-IX, zweimal in fachsprachen X-XVIII, einmal in fachsprachen XIX-XXVII und zweimal in fachsprachen XXVIII-XXXVI. Diese Erklärung zum Format ısechsmal vier` mag zur Illustration der oben erwähnten Koinzidenzen dienen. 
biges Lyrisches Ich oder das Gedicht selbst gemeint ist, ist unerheblich, weil es auf dasselbe hinausläuft. Das Vorbild, das - von wem auch immer - nachgeahmt werden will, ist in jedem Fall Hans Thill - oder überhaupt ein Vorbild, für das der Name Hans Thill um des Reimes willen bloß stellvertretend steht (denn inwiefern die Aussage zutrifft, wäre nun ebenfalls zu prüfen). Aber unabhängig davon, macht diese Aussage doch deutlich, worum es letztlich - mit einem ironischen Augenzwinkern gegen Kreativitätsrhetorik - gehen könnte: Wiederholbarkeit.

Fachsprachen erscheinen je - mehr oder weniger - einheitlich. Sofern sie in fachsprachen auftreten, kann die Erscheinungsweise natürlich willkürlich sein. Aber ehrlich gesagt, einen guten Anteil Willkür tragen auch die Erscheinungsweisen der Fachsprachen in sich. Diese Einheitlichkeit kann vorderhand also einen Ausgangspunkt bieten, sich mit Erscheinungsweisen zu beschäftigen, die im Voraus - ob willkürlich oder nicht - festgelegt worden sind. Auf die Textproduktion jedenfalls hat das entscheidende Auswirkungen. Und ich möchte im Weiteren nicht mehr von Erscheinungsweisen, sondern von Form (১typographisches Dispositiv $\iota$ ) sprechen. fachsprachen haben eine bestimmte Form, die rein phänomenologisch recht eindeutig beschrieben werden kann. Eine Form, die keinesfalls auf den Vers im herkömmlichen Sinn verzichtet, sondern aufgrund ihrer Wiederholbarkeit eben auf den - paradoxen - >freien ` Vers. Besonders deutlich zeigt sich dieser Umstand, dass Fachsprachen eine bestimmte und dadurch wiederholbare Form haben müssen, anhand einer (wissenschaftlichen) Philosophie, die sich streng an der Logik orientiert. Ihr Inhalt lässt sich mittels formaler (!) Logik im Handumdrehen schematisieren, beispielsweise als `Modus Ponens`, der als argumentative Grundstruktur verstanden werden darf: $\mathrm{a} \rightarrow \mathrm{b}, \mathrm{a} \mathrm{F}$ b. Und Stolterfoht erwähnt in fachsprachen XXVIII-XXXVI, wie bereits gezeigt, nicht nur die Logik und ihre Tradition, sondern bildet neben dem Gleichheitszeichen (vgl. Stolterfoht 2009, 18) mit Verweis auf Frege, einem der Gründerväter der modernen Logik, ja auch dessen Behauptungszeichen ab (Stolterfoht 2009, 94), wonach im obigen Schema der Aussage b der Wahrheitswert `wahr $<$ zugesprochen wird.

Im Nachdenken über diesen Sachverhalt drängen sich mir zwei Komponenten auf, die aller Wahrscheinlichkeit nach die fachsprachen hinsichtlich Produktion und Produkt ausmachen: eine technische zur Einhaltung der vorgegebenen Form sowie eine erkenntnistheoretische, welche in einem ersten Schritt die Form als Erkenntnismittel generiert und in einem zweiten Schritt die Form (>Faktur८) mit neuen bzw. anderen Inhalten (>Information`) abgleicht. ${ }^{8}$ Die Form soll etwas über

8 Entgegen Zymners Feststellung, `Faktur und `Information`seien kaum voneinander zu trennen, scheint mir Stolterfohts Lyrik diese Untrennbarkeit zumindest in Frage zu stellen. Auf der ersten Ebene gibt es keinen zwingenden Grund für das spezifische stypographische Dispositiv`. 
den Inhalt zu Tage fördern, das (vielleicht) angemessen scheint, aber sicher nicht so ohne Weiteres an die Oberfläche gekommen und sichtbar geworden wäre. Meine Hoffnung in der Auseinandersetzung mit Lyrik, wie Stolterfoht sie präsentiert, beruht natürlich im Allgemeinen auf der allfälligen Möglichkeit, eine a priorische Setzung ausmachen zu können, welche die Form immanent notwendig festlegt, noch bevor das erste Gedicht mit dieser Form erscheint. Aber selbst wenn dies nicht möglich ist - und bei Stolterfohts fachsprachen ist das mit großer Sicherheit nicht ohne Weiteres möglich ${ }^{9}$-, wird eine Form, ist sie einmal zum Prinzip erhoben, unausweichlich das Material strukturieren, ob sie nun von Anfang an begründet war oder sich erst im Nachhinein als nützlich erwiesen hat. Sie wird jedenfalls nachträglich das Material so ordnen, dass nur bestimmte Dinge erkannt oder verstanden werden können - andere wiederum werden ausgeschlossen. Rein quantitativ lässt sich das leicht nachvollziehen. Es passt nur so und so viel Sprachmaterial (`Umfang`) in eine vorgegebene Form. Das übrige Sprachmaterial ist ausgeschlossen und damit auch die ihm eigentümliche Erkenntnis. Form funktioniert so in der Lyrik vielleicht ganz im Sinn von Foucaults Diskursordnung. Während die Disziplin im wissenschaftlichen Diskurs jedoch vor allem regulativ wirkt, zeigt sie in der Lyrik ihr produktives Potenzial.

Und auf einer zweiten Ebene erweisen sich die Gründe aufgrund einer Logik der Serialität als unabhängig von der jeweiligen »Information«.

9 Im Fall von Stolterfohts fachsprachen liegt die a priorische Setzung also weniger in der jeweiligen Form des Gedichts als vielmehr im Konzept der fachsprachen selbst. Die Frage nach dieser a priorischen Setzung (unter anderem bei den Gedichten Stolterfohts) ist Ausgangspunkt meiner Dissertation. Zum Zeitpunkt der Niederschrift dieses Artikels waren meine Untersuchungen noch im Anfangsstadium. Dies unter anderem erklärt, weshalb die Stoßrichtung zwar schon gegeben und in diesem Artikel dargelegt ist, jedoch die Resultate noch wenig spezifisch sind. Allerdings lässt sich (mittlerweile ist das Kapitel zu Stolterfoht nämlich geschrieben) an dieser Stelle festhalten: Im Fall von Stolterfohts fachsprachen kehrt sich der Begründungszusammenhang um. Die spezifische Darstellung (১typographisches Dispositiv`) der fachsprachen lässt sich nicht a priorisch eruieren, allerdings erzeugt die konsequente Durchhaltung dieser Darstellung (`typographisches Dispositiv ) einen Effekt, der mit der Zeit einen reproduktiven Charakter annimmt, sodass die fachsprachen als lyrische Population beschrieben werden können. Einer Übersetzung Stolterfohts des Gedichts "winning his way« von Edith Stein folgend lässt sich das fachsprachen-Projekt in der Frage fassen, wie man seine art gewinnt. >Art ist dabei in einem dreifachen Sinn zu verstehen. Nicht nur geht es um die eigene Kunst (englisch: sart ‘), sondern darum, mit dieser Kunst - sowohl als Werk wie auch als gut eingeübte künstlerische Praxis - die eigene Art (biologische Population: Mensch) zu gewinnen, indem das einzelne Gedicht selbst wiederum als Individum einer Sepzies von Kunst (lyrische Population: fachsprachen) begriffen wird, deren Hauptmerkmal aufgrund der gut eingeübten künstlerischen Praxis in ihrer zuverlässigen Reproduzierbarkeit liegt. Der vorliegende Artikel kann nur den Aspekt Unabhängigkeit der fachsprachen von einer einzelnen sprechenden/schreibenden Instanz (`Adressant $`$ ) - sei diese nun der Autor oder ein lyrisches Ich -zeigen. 
Dieser Relativität von Erkenntnis sind wir unbemerkt schon lange vorher begegnet, als Sapir und Whorf in Schorfheide Hochzeit hielten. Die Sapir-WhorfHypothese (aufgestellt vom Linguisten und Ethnologen Edward Sapir in Zusammenarbeit mit seinem Studenten Benjamin Lee Whorf) postulierte Anfang des zwanzigsten Jahrhunderts ein linguistisches Relativitätsprinzip, wonach eine bestimmte Sprache sich auf die Welterfahrung der betreffenden Sprachgemeinschaft auswirkt, wie ich der Stanford Encyclopedia of Philosophy entnehme (vgl. Baghramian und Carter 2016). Diese unter anderem anhand von Untersuchungen der Hopi-Sprache in den USA aufgestellte Relativitätshypothese lässt sich meines Erachtens leicht und sinnvoll auf das Verhältnis von Fachsprachen übertragen. Dass Sapir und Whorf allerdings in Schorfheide, einem Waldgebiet nordöstlich von Berlin, Hochzeit gehalten haben sollen, steht nicht in besagter Enzyklopädie - und ist verständlicherweise nicht zu wissen. Schließlich waren die beiden Amerikaner und nie in Schorfheide, so lässt sich annehmen. Heinrich Zille hingegen, der deutsche Grafiker, Maler und Fotograf, ehelichte die Fürstenwalderin Hulda Frieske und wird wohl in Fürstenwalde Hochzeit gehalten haben.

Es handelt sich bei »deklaration des wust«, erinnert sei an Austins Einschränkung, also sowohl um ein Gedicht als auch um einen Sprach-Witz mittels Binnenreimen. Der Wust des - gewissermaßen - Gewussten in diesem Gedicht ist beachtlich. Und der Hinweis auf Sapir und Whorf zeigt auch, dass das Zitieren eines linguistischen Wörterbuchs am Anfang nur scheinbar voreilig war.

Gedichte als fachsprachen sind also, wie Stolterfoht im Gedicht »aggregate V« sagt, »sanftem zwang" (Stolterfoht 2013, 203) unterworfen und üben diesen wiederum aus, wenn wir an ihren Aus- und Einschlusscharakter denken. Der sanfte Zwang, und gemeinhin steht Zwang in ästhetischen Belangen heute ganz allgemein nicht besonders hoch im Kurs, ${ }^{10}$ zeitigt jedoch - zumindest im Fall von Stolterfohts

10 Dieser eigentümliche »sanfte zwang « macht hellhörig. Vor dem Hintergrund eines demokratischen Anspruchs klingt unweigerlich der ebenso »eigentümlich zwanglose Zwang des besseren Arguments« an, den Jürgen Habermas in seiner Theorie des kommunikativen Handelns anführt. Die Rede vom Zwang wirft nicht nur ein irritierendes Licht auf die gegenwärtigen demokratischen Verhältnisse westlichen Zuschnitts bzw. die mit ihnen implizit verbundenen Erwartungen individueller Freiheit, sondern auch auf die oft in Anschlag gebrachte Freiheit künstlerischen Schaffens. Vielleicht ist in beiden Fällen zu Recht eine Kluft zwischen Selbstverständnis und Gegebenheiten zu vermuten. Die Forderung nach der Rationalität eines demokratischen Diskurses verstanden als »handlungskoordinierender Mechanismus« (Habermas 1995, 148) blendet aus, dass Demokratie, ihrer eigenen Logik gehorchend, möglicherweise darauf hinauslaufen könnte, gerade das Handeln zu unterlassen: das endlose Konstatieren des Dissenses statt das Erreichen eines handlungsleitenden Konsenses - und Dissens ist dabei noch das falsche Wort, weil es nie um das Erreichen eines Konsenses ging. Darin scheint für die Politik Hannah Arendts zutiefst menschlicher Begriff des Handelns bzw. vor allem des Sprechens auf, wie sie ihn unter anderem 
fachsprachen - beachtliche Resultate und ich möchte in diesem Zusammenhang auf ein Diktum von Michael Lentz aus seiner Frankfurter Poetikvorlesung von 2013 hinweisen, dass nämlich ein »regelpoetisch angefertigtes Prokrustesbett allemal überraschendere, scharfsinnigere, rätselhaftere Ergebnisse zeitigt als die pure Not literarischen Produzierens." (Lentz 2013, 22) Stolterfoht selbst legt mit dem im vierten Fachsprachenband als Zitat angeführten Kunstwort »prokrustik» (Stolterfoht 2009, 43) diese Verbindung nahe. Und in diesem Licht ergibt Adornos Satz aus der Ästhetischen Theorie Sinn: »Im Mythos von Prokrustes wird etwas von der philosophischen Urgeschichte der Kunst erzählt.« (Adorno 1973, 217)

Nicht von ungefähr - und wohl auch im Sinne der von Lentz angeführten Regelpoetik - ist also am Anfang des Gedichts »deklaration des wust « vom »lyrik-/ programm « die Rede. Insofern ist die Frage »Wer spricht im Gedicht? « - und Stolterfoht stellt sie hinterhältigerweise und völlig unvermittelt auch: »wer spricht?« (Stolterfoht 2009, 93) - aus Sicht der fachsprachen eher spitzbübisch grinsend. Denn es sind die fachsprachen selbst, die gesprochen werden - auch wenn die fachsprachen natürlich darauf angewiesen sind, dass jemand sie spricht. Aber das gilt für das Sprechen überhaupt: Die Sprache macht den Sprecher und der Sprecher macht die Sprache. Die Frage ist nur, was jeweils im Vordergrund steht. Wenigstens dies aufzuzeigen, dazu scheinen die fachsprachen doch recht geeignet. Es sind also selbstverständlich die fachsprachen, die gesprochen werden von niemandem, wenn sie denn demokratisch und erkennend sind. Die paradoxe Konsequenz angesichts dieser Lyrik: Niemand spricht von nichts. ${ }^{11}$ Und auch der inhärente Zwang, der sich doch immer gegen Einzelnes - oder mit Celans Büchner gesprochen: die »Kreatur« (Celan 1999, 5) - richtet, macht noch einmal deutlich,

in der Vita activa (Arendt 2007, 213-251) und in den posthum erschienen Fragmenten Was ist Politik? (Arendt 2003, 35-80) anhand der griechischen Poliserfahrung entfaltet. Dieser Begriff des Sprechens als genuine politische Tätigkeit und Verwirklichung der höchsten menschlichen Möglichkeit muss zwar vor dem Hintergrund der realen Lebenswelt und insbesondere auch aus heutiger Sicht, dessen ist sich Arendt durchaus bewusst, eigenartig apolitisch erscheinen - dennoch mag er unter heutigen Umständen als, vielleicht utopisches, Korrektiv wirken. Das freie Sprechen, das die Politik ist, hat in der Zuspitzung Arendts nämlich vor allem den Zweck der Selbstoffenbarung der sprechenden Personen. - Für die Lyrik erhellt das möglicherweise, weit über die fachsprachen hinausweisend, Stolterfohts anvisierten »Realismus zweiter Ordnung" (Stolterfoht 2010, 33), wo der Zweck lyrischer Sprache wiederum deren Selbstgenügsamkeit und insofern völlig jenseits jedes erdenklichen Erkenntnisinteresses auch deren Selbstoffenbarung in gewissem Sinn unabhängig von der dichtenden Person und gerade deshalb vielleicht wieder in höchstem Maß mit der dichtenden Person verbunden - ist.

11 Stolterfoht bekräftigt bestimmte »unhintergehbare erkenntniskritische Grundeinsichten, die es verbieten, in Gedichten süber oder ıvon` etwas zu schreiben, etwas ızum Ausdruck zu bringen`, womöglich auch noch raus sich selbst zu schöpfen`« (Stolterfoht 2015, 15). 
dass in den auf den ersten Blick durchaus rätselhaften fachsprachen das lyrische Ich als konkrete Person bzw. Instanz wohl eine untergeordnete Rolle spielt - auch wenn dieser Zwang, sofern er sanft und doch unausweichlich ist, mit aller Wahrscheinlichkeit erst den Erscheinungsraum von Einzelnem konstituiert.

\section{Literaturverzeichnis}

Adorno, Theodor W. Ästhetische Theorie. Frankfurt a. M.: Suhrkamp, 1973.

Arendt, Hannah. Was ist Politik? Hg. Ursula Ludz. München: Piper Verlag, 2003.

Arendt, Hannah. Vita activa. Fünfte Auflage. München: Piper Verlag, 2007.

Austin, John L. How to do things with words. Hg. J. O. Urmson und Marina Sbisa. London: Oxford Univ. Press, 1976.

Baghramian, Maria und J. Adam Carter. »Relativism«. The Stanford Encyclopedia of Philosophy (Spring 2016 Edition). Hg. Edward N. Zalta. http://plato.stanford.edu/archives/spr2016/ entries/relativism/. (15. August 2016).

Burdorf, Dieter. Einführung in die Gedichtanalyse. 2. Aufl. Stuttgart: Metzler 1997.

Celan, Paul. Der Meridian - Endfassung Vorstufen Materialien. Hg. Bernhard Böschenstein und Heino Schmull. Frankfurt a. M.: Suhrkamp, 1999.

Celan, Paul. Die Gedichte. Hg. Barbara Wiedemann. Frankfurt a. M.: Suhrkamp, 2005.

Foucault, Michel. Die Ordnung des Diskurses. Übers. Walter Seitter. Frankfurt a. M.: Fischer, 1991.

Fricke, Harald und Peter Stocker. »Lyrisches Ich «. Reallexikon der deutschen Literaturwissenschaft, 2. Bd. Hg. Klaus Weimar, Georg Braungart, Harald Fricke, Klaus Grubmüller, Jan-Dirk Müller und Friedrich Vollhardt. Berlin: de Gruyter, 2000. 509-511.

Hegel, G. W. F. Phänomenologie des Geistes - Werke 3. Hg. Eva Moldenhauer und Karl Markus Michel. Frankfurt a. M.: Suhrkamp, 1986.

Hölderlin, Friedrich. „Der Winkel von Hahrdt«. Frankfurter Hölderlinausgabe Bd. 8. Hg.

D. E. Sattler. Frankfurt a. M.: Stroemfeld Verlag 2000. 758.

Lentz, Michael. ATMEN ORDNUNG ABGRUND. Frankfurt a. M.: Fischer, 2013.

Lewandowski, Theodor (Hg.). Linguistisches Wörterbuch, 5. Aufl. Heidelberg: Quelle \& Meyer, 1990.

Stolterfoht, Ulf. fachsprachen I-IX. Basel: Engeler, 2005.

Stolterfoht, Ulf. fachsprachen XIX-XXVII. Basel: Engeler, 2005.

Stolterfoht, Ulf. fachsprachen X-XVIII. Basel: Engeler, 2008.

Stolterfoht Ulf. fachsprachen XXVIII-XXXVI. Basel: Engeler, 2009.

Stolterfoht, Ulf. Ammengspräche. Hg. Urs Engeler. Berlin: Roughbooks, 2010.

Stolterfoht, Ulf. »aggregate V«. Jahrbuch der Lyrik 2013. Hg. Christoph Buchwald und Jan Wagner. München: Deutsche Verlags-Anstalt, 2013: 203.

Stolterfoht, Ulf. Wurlitzer Jukebox Lyric FL - Münchner Reden zur Poesie. München: Stiftung Lyrik Kabinett München, 2015. 